\title{
Programa de apoio matricial em cardiologia: qualificação e diálogo com profissionais da atenção primária
}

\section{Matrix support program in cardiology: qualification and dialogue with primary care professionals}

\author{
Clovis Hoepfner \\ Mestre em Saúde e Meio Ambiente. Docente do Departamento \\ de Medicina da Universidade da Região de Joinville e Médico \\ Cardiologista do Hospital Municipal São José. \\ Endereço: Rua Orestes Guimarães, 422, Centro, CEP 89204-060, \\ Joinville, SC, Brasil. \\ E-mail: hoepfnerळcardiol.br \\ Selma Cristina Franco \\ Doutora em Saúde da Criança e do Adolescente. Docente do De- \\ partamento de Medicina da Universidade da Região de Joinville. \\ Endereço: Rua Otto Boehm, 80I, apto 40I, CEP 8920I-700, Joinville, \\ SC, Brasil. \\ E-mail: scfranco®terra.com.br

\section{Rosemeri Aparecida Maciel} \\ Especialista em Saúde da Família. Enfermeira responsável técnica \\ pelo Programa de Hipertensão Arterial e Diabetes Melito da Se- \\ cretaria Municipal da Saúde de Joinville. \\ Endereço: Rua Porto Alegre, 63, CEP 89207-680, Joinville, SC, Brasil. \\ E-mail: macielrosemeriळgmail.com

\section{Angela Maria da Silva Hoepfner} \\ Mestre em Psicologia. Psicóloga na Atenção Primária em Saúde da \\ Secretaria Municipal de Saúde e Docente Convidada da Escola de \\ Saúde Pública de Santa Catarina. \\ Endereço: Rua Orestes Guimarães, 422, apto 90I, CEP 89204-060, \\ Joinville, SC, Brasil. \\ E-mail: angelahoepfnerœhotmail.com
}

\section{Resumo}

O apoio matricial é uma estratégia potente e inovadora para enfrentar o desafio de reorganizar e qualificar o processo assistencial das doenças crônicas não transmissíveis no Sistema Único de Saúde (SUS). Este artigo relata a implantação do Programa de Apoio Matricial em Cardiologia na rede básica de saúde de um município na Região Sul do Brasil e seus resultados iniciais. Trata-se de um estudo de pesquisa-ação no qual os pesquisadores em campo foram um cardiologista e uma enfermeira que participaram ativamente do delineamento do Programa de Apoio Matricial em Cardiologia. O material empírico foi constituído por anotações de campo, registros da Central de Regulação e depoimentos dos principais agentes envolvidos na implantação e realização do apoio matricial, obtidos em entrevistas semiestruturadas e reuniões. 0 programa foi implantado em 2010, em todas as 56 unidades básicas de saúde com os objetivos de expandir a educação permanente para os profissionais da rede e capacitar as equipes de saúde para a resolução de problemas cardiológicos que demandem menor densidade tecnológica. Os resultados evidenciam maior resolubilidade dos médicos da atenção primária, com consequente redução e qualificação dos encaminhamentos para os cardiologistas, acesso mais rápido aos cardiologistas e aos exames, além de importante redução da fila dessa especialidade. Propõe-se a continuidade desse programa, sua reprodução em outros locais e incorporação de outros profissionais que atuam nas equipes multidisciplinares da atenção primária.

Palavras-chave: Educação Continuada; Apoio ao Desenvolvimento de Recursos Humanos; Apoio Matricial; Cardiologia; Atenção Primária à Saúde. 


\section{Abstract}

Matrix support is a powerful and innovative strategy to face the challenge of reorganizing and qualifying the clinical process of chronic non-communicable diseases in the Brazilian National Health System. This article reports the deployment of the Matrix Support Program in Cardiology in primary health care services in a town in southern Brazil and its initial results. This is an action research study in which the field researchers were a cardiologist and a nurse who actively participated in designing the Matrix Support Program in Cardiology. The empirical material consisted of field notes, records from the Regulation Center, and accounts by key players involved in the deployment and execution of matrix support, obtained by means of semi-structured interviews and meetings. The program was deployed in 2010, in all of the 56 primary health centers aiming both to increase continuing education for professionals in the network and train the health teams to solve cardiologic problems requiring less technological resources. The results show better ability to solve problems on the part of the primary care physicians, with consequent decreased and qualified referrals to cardiologists, faster access to cardiologists and examinations, in addition to a significantly shorter queue for this specialty. We propose to continue this program, replicate it in other locations, and incorporate other professionals working in the multidisciplinary teams of primary care.

Keywords: Education Continuing; Training Support; Matrix Support; Cardiology; Primary Health Care.

\section{Introdução}

\section{As Doenças Cardiovasculares e a Reorganização dos Processos de Trabalho em Saúde}

As doenças crônicas não transmissíveis (DCNTs), dentre elas as doenças cardiovasculares (DCVs), ocasionam impacto significativo no planejamento e no custo das ações sanitárias efetuadas pelo Sistema Único de Saúde (SUS) (Brasil, 2006). Além do investimento na assistência, faz-se necessário o desenvolvimento de ferramentas que permitam melhorar a abordagem destas doenças e seus fatores de risco. A literatura científica na área e as experiências de outros países destacam que as tecnologias mais efetivas para a redução da carga de DCNTs são aquelas que trabalham com os fatores determinantes e condicionantes da saúde individual e coletiva, elaborando políticas públicas orientadas pela ótica da integralidade e adequadas à realidade de cada região (Brasil, 2006).

A elevada morbimortalidade das DCVs coloca-as como prioritárias dentre as políticas de saúde em nosso país. Tais doenças requerem medicamentos de uso continuado e mudanças nos hábitos de vida, características que demandam um processo contínuo de atenção e, nos momentos de sofrimento agudo, uma assistência imediata utilizando as tecnologias exigidas para cada condição clínica (Brasil, 2006).

Para integrar os diversos níveis de atenção da rede de atendimento em saúde, garantindo o acesso e a resolutividade dos serviços, são necessários rearranjos nos processos de trabalho, as linhas de cuidados, envolvendo todos os níveis assistenciais em uma trabalho interdisciplinar, visando a integralidade do cuidado. Trata-se de uma proposta de mudança de paradigma: do foco biomédico, centrado na doença e na prática médica curativa para uma visão centrada no paciente e suas necessidades, com responsabilidade compartilhada pela equipe multidisciplinar e criação de vínculo terapêutico (Brasil, 2006).

Embasadas assim por uma clínica ampliada em que as ações de proteção, promoção, vigilância, assistência e reabilitação são voltadas para as especificidades de grupos e indivíduos, as linhas de cuidado permitem não só a condução oportuna dos pacientes pelas diversas possibilidades diagnósticas 
e terapêuticas, mas também uma visão global das suas condições de vida (Brasil, 2006).

Para que esta mudança de modelo se efetive, são imprescindíveis o apoio e o investimento dos gestores para vencer dificuldades na estrutura dos serviços de saúde e ampliar as possibilidades de realizar esta proposta de trabalho com a interação dialógica entre os trabalhadores da saúde e destes com os usuários (Brasil, 2006).

O rearranjo dos processos de trabalho em linhas de cuidado demanda uma tecnologia organizacional complementar àquela prevista em sistemas hierarquizados, a saber: mecanismos de referência e contrarreferência, protocolos e centros de regulação. Chamada de apoio matricial e proposta por Campos (1999), recomenda a reorganização dos processos de trabalho por meio da composição de equipes básicas de referência, multidisciplinares, que atuariam em distintos serviços de saúde, tendo uma população a elas adscrita e que receberiam apoio especializado matricial de profissionais, conforme as necessidades assistenciais e os limites de intervenção identificados pelas equipes de referência.

Desse modo, o apoio matricial pretende oferecer tanto retaguarda assistencial quanto suporte técnico-pedagógico às equipes da Atenção Primária à Saúde (APS), qualificando-as e tornando-as mais resolutivas para atuarem nos territórios junto aos usuários e, assim, fortalecer o vínculo entre os profissionais envolvidos (o generalista, o enfermeiro, o especialista e outros) por meio de um trabalho coletivo e enriquecedor para todos. Para isso, há necessidade da construção compartilhada de diretrizes clínicas e sanitárias, o que compreende os chamados projetos terapêuticos singulares (PTSs), elaborados pelos componentes de uma equipe de referência e os especialistas que oferecem apoio matricial. Essas diretrizes devem prever critérios para solicitar o apoio sempre que necessário e definir o espectro de responsabilidades tanto dos diferentes integrantes da equipe de referência quanto dos apoiadores matriciais (Campos e Domitti, 2007; Campos e Cunha, 2011).

Nos sistemas de saúde organizados de forma hierarquizada e regionalizada, como é o caso do Brasil, a APS tem a responsabilidade de realizar um conjunto de ações de saúde, no âmbito individual e coletivo, compreendidas como de produção de saúde, isto é, abrangendo a promoção e a proteção, a prevenção de agravos, o diagnóstico e o tratamento, a reabilitação e a manutenção da saúde. A APS é o nível do sistema de saúde que constitui a entrada preferencial para as novas necessidades e problemas de saúde da população, fornecendo atenção para as pessoas no decorrer do tempo e atuando sobre as condições mais prevalentes, além de coordenar ou integrar a atenção obtida em outros pontos ou por terceiros (Cotta e col., 2006). Neste modelo, confere-se às equipes da APS, incluindo os médicos, enfermeiros, técnicos de enfermagem e agentes comunitários de saúde, grande importância nos cuidados aos portadores de DCV.

\section{A Qualificação dos Profissionais para Atuar com as DCV}

Para a qualificação e a atualização dos profissionais de saúde, são utilizados programas de educação permanente em serviço. Na área de Cardiologia, as diversas diretrizes da Sociedade Brasileira de Cardiologia, entre outras, são utilizadas como documentos de atualização. Todavia, o conhecimento do conteúdo das diretrizes por si só não representa uma garantia do seu uso apropriado (Mion e col., 2007; Milchak e col., 2004; Lemos e col., 2009). Milchak e colaboradores (2004), em avaliação da literatura acerca da aderência dos médicos às diretrizes em diversos países, relataram a existência de substanciais lacunas entre o desenvolvimento dos documentos, a disseminação e a sua implantação. Segundo o autor, vários pesquisadores concluíram que as diretrizes não modificam de forma consistente o comportamento dos profissionais. No Brasil, Lemos e colaboradores (2009) mostraram que muitas das recomendações das diretrizes não são seguidas pelos médicos da APS.

As estratégias de educação médica permanente foram revisadas e classificadas pelo grau de eficácia por Davis e colaboradores (1995) em metanálise que incluiu 99 estudos. Os métodos considerados mais eficazes foram as intervenções sistemáticas baseadas na prática cotidiana. O Programa Canadense de Educação em Hipertensão (CHEP), de 1999, continuamente avaliado e aperfeiçoado, é um modelo que tem sido recomendado para outros países (McAlister, 2006). 0 programa utiliza nume- 
rosas ferramentas para educação permanente dos profissionais da saúde, incluindo workshops com lideranças locais e nacionais, capacitação de formadores locais, periódicas atualizações publicadas em revistas científicas, livros de bolso, distribuição frequente de resumos e pôsteres, além do site da Sociedade Canadense de Hipertensão. Onysko e colaboradores (2006) e Campbell e colaboradores (2009) ao estudarem o CHEP, mostraram o grande incremento no diagnóstico e no tratamento da doença e a redução das complicações dela decorrentes, após sua implantação.

Assim, a literatura tem apontado a importância de se investir em programas de educação permanente que, além do aspecto formativo, consigam modificar a atuação dos profissionais visando a melhor qualidade assistencial.

Com este intuito, a Secretaria Municipal de Saúde (SMS) de Joinville desenvolve programas de educação permanente em Cardiologia desde 1995, incluindo a confecção de protocolos e diretrizes de hipertensão arterial e cursos de atualização para médicos e enfermeiros (Joinville, 2007). Apesar dos esforços empreendidos, estes programas, concebidos dentro de um modelo tradicional e pouco participativo, foram mostrando, ao longo dos anos, resultados primordialmente informativos e sem impacto na mudança de comportamento dos profissionais, conforme apontam pesquisas realizadas em 2008 e 2009 sobre conhecimentos e práticas em hipertensão arterial entre os profissionais enfermeiros e médicos da atenção primária (Hoepfner, 2010). Ao mesmo tempo foi realizada uma avaliação acerca do controle da hipertensão arterial, da presença de fatores de risco aterosclerótico e de complicações associadas à doença (Hoepfner e Franco, 2010), que constatou baixa utilização do protocolo e dos instrumentos de coleta e aferição de dados clínicos dos pacientes, várias distorções no acompanhamento da hipertensão arterial e das cardiopatias e controle insuficiente da doença. Além disso, ao longo dos últimos anos, identificou-se um acúmulo progressivo de pacientes nas filas de espera aguardando consultas especializadas em Cardiologia, ofertadas em ambulatórios próprios e pelos dois hospitais públicos, um deles municipal. Dentro da lógica focada na realização de procedimentos, no período de 2008 a 2010, apesar do aumento na oferta de consultas, o tempo de espera pelo atendimento do cardiologista passou de um para três anos, seguido por uma espera de vários meses para os exames complementares, como o ecocardiograma e o teste ergométrico. A fila de espera chegou a acumular, em janeiro de 2010, mais de onze mil pacientes. Esta situação evidenciou o esgotamento do modelo proposto e foi considerada crítica, motivando uma intervenção da gestão municipal. Após consulta pública aos profissionais da saúde, foi revisada e ampliada a diretriz de hipertensão arterial (Joinville, 2010), e foram propiciadas diversas atividades de educação permanente na área da Cardiologia. Também ocorreu uma reorganização no processo de atenção aos pacientes portadores de doenças cardiovasculares, com a introdução do apoio matricial. $\mathrm{O}$ objetivo principal deste estudo é relatar a implantação do Programa de Apoio Matricial em Cardiologia e seus resultados iniciais.

\section{Metodologia}

Trata-se de um estudo de pesquisa-ação que teve como objeto o Programa de Apoio Matricial em Cardiologia implantado na SMS de Joinville em fevereiro de 2010, sendo que os resultados aqui apresentados referem-se aos primeiros trinta meses de sua existência. A pesquisa-ação, conforme proposta por Thiollent (2011) é um

tipo de pesquisa social com base empírica que é concebida e realizada em estreita associação com uma ação ou com a resolução de um problema coletivo e no qual os pesquisadores e os participantes representativos da situação estão envolvidos de modo cooperativo e participativo (p. 14).

Os pesquisadores em campo foram um cardiologista e uma enfermeira que participaram ativamente do delineamento do Programa de Apoio Matricial em Cardiologia e da presente pesquisa que ocorreu pari passu à sua implantação. Os demais participantes foram os principais agentes envolvidos para implantar e realizar o apoio matricial, cujos depoimentos aqui relatados permitem analisá-lo a partir de diferentes olhares. São eles: o gestor municipal de saúde, diretores do hospital municipal, profissionais responsáveis pelo gerenciamento da rede de APS, médicos cardiologistas que realizam o 
apoio matricial, profissionais das equipes básicas de referência que atuam na APS e outros cardiologistas que atuam nos serviços públicos municipais.

Participaram do programa todas as 56 unidades de APS existentes no município, das quais 36 organizam-se segundo o modelo de Estratégia de Saúde da Família (ESF), 14 segundo a Estratégia de Agentes Comunitários de Saúde (EACS) e 6 segundo o modelo convencional.

O estudo utilizou como material empírico os registros da Central de Regulação da SMS relacionados com a especialidade de Cardiologia do adulto, anotações efetuadas em diário de campo pelos pesquisadores durante a execução das atividades de apoio matricial e depoimentos de diversos profissionais de saúde obtidos em entrevistas semiestruturadas e em reuniões. Os resultados do programa aqui apresentados foram categorizados em: descrição da implantação do programa, impacto sobre a organização da assistência e impacto sobre a formação dos profissionais.

\section{Resultados}

\section{A implantação do Programa de Apoio Matricial}

O Programa de Apoio Matricial em Cardiologia foi apresentado e debatido com atores ligados à gestão municipal da saúde e à assistência em cardiologia nos diversos níveis de atenção. Nesses encontros, acontecidos no final de 2009 e início de 2010, buscou-se consenso sobre os objetivos, meios e resultados pretendidos, por meio de um conjunto de atividades que incluíram a capacitação in loco, ou seja, nas unidades básicas de saúde, e estabeleceu-se que as atividades centrais seriam os atendimentos conjuntos entre o cardiologista e o médico da equipe de referência, com discussões de casos reais e de diversos temas de Cardiologia, além de orientações acerca da linha guia de hipertensão. Tanto aqui como ao longo do processo, descobriu-se que poucos profissionais tinham tido contato prévio com o método de trabalho do apoio matricial. Algumas unidades básicas de saúde apresentaram-se como voluntárias para iniciar o programa e participaram da confecção de um calendário de atividades. 0 apoio matricial foi planejado para ocorrer em dois dias semanais, sendo efetuado por um médico cardiologista e uma enfermeira do nível central da Secretaria Municipal de Saúde que se deslocavam até as unidades. A retaguarda assistencial contou com dois cardiologistas, incluindo o matriciador. A agenda dos dois cardiologistas foi modificada para incluir vagas semanais exclusivas do apoio matricial, sem aumentar o número total de atendimentos. Essa reserva técnica foi destinada aos pacientes selecionados para atendimento prioritário. Essa seleção ocorreu durante as discussões dos casos nas unidades, mediante acordo entre a equipe de matriciamento e os profissionais da APS. Antes dos encontros, as equipes da APS separavam os prontuários dos pacientes e os formulários de encaminhamento ao cardiologista em dois grupos, dando prioridade aos pacientes julgados em situação de risco. A discussão dos casos propiciou identificar os temas relevantes para serem abordados nas atividades de educação em serviço. Inicialmente propôs-se a participação obrigatória para os médicos e os enfermeiros nas discussões de casos, deixando a critério deles a participação dos técnicos de enfermagem, agentes comunitários de saúde (ACSs) e outros profissionais da equipe, com o cuidado de se preservar os diagnósticos clínicos dos pacientes, por questões éticas.

\section{Impacto sobre a assistência}

Na primeira etapa, entre fevereiro e abril de 2010, o programa envolveu 11 unidades em nove semanas, sendo discutidos com os médicos e enfermeiros os casos de 247 pacientes encaminhados ao cardiologista, dos quais foram selecionados 83 pacientes para agendamento prioritário e 12 para agendamento com especialistas de outras áreas. Na reunião de avaliação, realizada ao final deste período, incluindo representantes das unidades matriciadas e outros, considerou-se a metodologia de trabalho e os resultados satisfatórios, e decidiu-se dar continuidade ao programa. Dessa forma, entre fevereiro e dezembro de 2010, o programa abrangeu 41 das 56 unidades, com reuniões de avaliação periódicas. Dos 101 médicos, 90 enfermeiros e 224 técnicos de enfermagem ativos na APS nesse período, foram envolvidos 51 médicos, 47 enfermeiros, 31 técnicos e 48 alunos do curso de Medicina. No período de onze meses foram analisados 1.342 encaminhamentos ao especialista, dos quais foram selecionados $493(36,7 \%)$ pacientes 
para atendimento prioritário, em prazo máximo de dois meses, e foi decidida a exclusão de 298 usuários da fila de espera (22,2\%). Em setembro de 2010, mais dois cardiologistas concursados foram contratados e incluídos no apoio matricial, em horários diferenciados, adicionando oito dias de retaguarda assistencial e dois dias de apoio semanal, permitindo alcançar mais 14 unidades, com cobertura da totalidade da rede básica municipal. Deles apenas citamos as UBSs matriciadas, pois os demais dados tiveram registro parcial, impossibilitando a sua inclusão nos resultados.

Em 2011, em virtude de uma prolongada greve dos funcionários públicos, apenas quinze unidades foram matriciadas pela equipe original. Outras vinte e três reuniões aconteceram com os outros cardiologistas. No primeiro semestre de 2012, foram matriciadas 20 UBSs. Ao final de trinta meses, foram geradas 437 exclusões da fila de espera de consulta com cardiologista, mediante consenso entre matriciador e equipe de referência. Os motivos foram usuários cujos problemas já haviam sido solucionados pela equipe de referência, os que obtiveram respostas em atendimento especializado privado e aqueles que a equipe da APS reassumiu após participar do atendimento conjunto e das discussões de casos com os matriciadores.

O impacto sobre a fila foi muito relevante ao final de dois anos, passando de 11.180 para 3.739 pacientes, apesar das novas demandas geradas neste período. Identifica-se um predomínio de retornos, representando $80 \%$ da demanda. A quantidade de usuários remanescentes é a menor dos últimos cinco anos e a maioria deles poderá ser atendida no prazo de até três meses. Vale ressaltar que, mesmo sem considerar os outros resultados positivos do programa em termos de formação dos profissionais, o grande número de pacientes que ainda permanecem na fila, por si só, já justifica sua continuidade.

\section{Impacto sobre a formação dos profissionais}

No primeiro ano do apoio matricial, foi dada prioridade para a avaliação dos encaminhamentos para os cardiologistas, mediante a sua análise conjunta, com os médicos e enfermeiros das unidades da referência. Tal atividade propiciou a discussão de numerosos temas da Cardiologia.
Observou-se que muitos dos encaminhamentos ao especialista se deram em virtude dos laudos dos exames complementares. Os eletrocardiogramas com os laudos de bloqueios divisionais do feixe de His, extrassístoles e anormalidades inespecíficas da repolarização ventricular estiveram entre os principais motivos para os encaminhamentos.

Outra causa frequente de solicitação de avaliação foram os laudos radiológicos de possível aumento do ventrículo esquerdo. Dor torácica atípica, a dificuldade de controlar os níveis da pressão arterial e a insegurança perante os portadores de doença coronariana, mesmo quando assintomáticos, também foram geradores de muitos dos encaminhamentos. A doença hipertensiva ocupou um amplo espaço nos encontros, especialmente a questão da adesão à terapêutica, as associações de medicamentos, a inércia terapêutica e a preocupação com as chamadas urgências ou "pseudourgências" hipertensivas. De 2010 para 2012, ocorreu redução na média de casos discutidos por unidade, de 32,7 para 12 e nos agendamentos prioritários de 12 para 3,4 usuários.

Os encontros também propiciaram a ausculta das queixas dos profissionais, sendo recorrente a reclamação da ausência da contrarreferência, de todas as especialidades. Também foram relatados problemas de estrutura dos serviços, como a falta crônica de equipamentos básicos como os esfigmomanômetros para obesos e crianças, a impossibilidade de solicitar os exames de eletrocardiografia de esforço e ecocardiografia, a elevada rotatividade de profissionais médicos e a falta de profissionais de endocrinologia e de nutrição e dietética foram muito frequentes.

Em algumas unidades, o apoio de acadêmicos de Medicina propiciou a avaliação da aferição da pressão arterial, rotineiramente executada pelos técnicos de enfermagem, constatando-se a técnica fora dos moldes recomendados pelas diretrizes. Em quatorze unidades (25\%) foi possível reunir-se com os profissionais da enfermagem para orientar a técnica adequada de aferição da pressão arterial e para discutir e orientar condutas no atendimento aos usuários hipertensos e cardiopatas. As maiores dúvidas destes profissionais estavam na conduta perante o hipertenso portador de sintomas inespecíficos, tais como cefaleia e tonturas, devido aos supos- 
tos riscos de complicações agudas da hipertensão, sendo frequentes os encaminhamentos de usuários aos serviços de pronto atendimento.

O endereço eletrônico dos apoiadores foi disponibilizado e tem sido utilizado pelos profissionais da APS para a discussão de situações clínicas mais complexas e para acelerar agendamentos. Artigos científicos e diretrizes nacionais e internacionais foram distribuídos ou disponibilizados eletronicamente.

Atualmente, o programa continua e outros profissionais da saúde, como os agentes comunitários, os odontólogos e os farmacêuticos tem sido incluídos nas reuniões desde janeiro de 2012.

\section{Discussão}

A aplicação dos conceitos do apoio matricial e equipes de referência vêm sendo recomendada pelo Ministério da Saúde (Brasil, 2010), sendo possível encontrar-se trabalhos sobre sua utilização por equipes de Saúde Mental em Natal, Capivari, Campinas e Fortaleza (Dimenstein e col., 2009; Arona, 2009; Figueiredo e Campos, 2009; Morais e Tanaka, 2012), todavia nenhum em Cardiologia. Em Joinville, a iniciativa de iniciar o programa foi rapidamente avalizada pelos gestores e bem recebida pelos profissionais da saúde, gerando ações imediatas. Diante da situação caótica das filas de espera, não houve uma discussão prolongada nem uma preparação dos participantes acerca do programa, como ocorreu noutros locais (Dimenstein e col., 2009; Arona, 2009; Figueiredo e Campos, 2009). A possibilidade de realizar um estudo piloto, restrito a algumas unidades, foi cogitada e descartada pelos gestores e pelos profissionais da APS. A grande demanda pelos especialistas foi o motivo para optar-se por estender o apoio matricial em Cardiologia a todas as unidades.

Embora tenham participado médicos, enfermeiros, estudantes de Medicina, profissionais de nível técnico e agentes comunitários de saúde, predominaram as reuniões com médicos e enfermeiros, profissionais centrais no modelo assistencial atual. A presença do médico nas discussões dos casos foi considerada imprescindível, uma vez que um dos objetivos do programa era conseguir vagas para o atendimento ambulatorial pelo cardiologista.
Aqui pode estar um problema decorrente da falta de discussão do programa, previamente, com os profissionais da APS, pois estas e outras atividades poderiam ter sido executadas com a participação dos demais membros das equipes de referência e os matriciadores. Nas falas dos entrevistados de Morais e Tanaka (2012), também fica evidente a maior participação de médicos e de enfermeiros no matriciamento, lamentando-se a falta de outros profissinais da equipe. Segundo estes autores, isto tira do apoio matricial a sua função estratégica de favorecer a reorganização do serviço e das práticas.

Com relação à visão dos profissionais a respeito do programa, um dos aspectos considerados mais positivos foi a revisão da condição clínica de cada paciente que aguardava na fila de espera, conforme relata um dos participantes: No apoio matricial, a gente faz uma limpa nos encaminhamentos, pois o posto descobre paciente aguardando há anos, alguns já falecidos e outros já resolvidos.

Ficou explícito que o seguimento de muitos dos usuários das unidades era insuficiente e que inexistia uma reavaliação periódica dos encaminhamentos. A busca pelos usuários, desencadeada pelo programa, revelou óbitos não comunicados, mudanças de domicílio e consultas já realizadas e não comunicadas pelo usuário. Um achado importante foi a constatação, nos prontuários, de que muitos pacientes estavam com a hipertensão arterial controlada sem a intervenção do cardiologista. Estes usuários foram imediatamente retirados da fila da cardiologia. Segundo depoimentos, a reavaliação dos encaminhamentos não urgentes, pelas equipes de referência, resultou em mais exclusões da fila. A diminuição da fila para o especialista agrada o gestor, sequioso por resultados numéricos positivos na sua gestão, expõe os sucessos das equipes da APS, antes invisíveis, fortalece o investimento nas ações de apoio matricial e reafirma as expectativas da Política Nacional de Humanização (PNH) (Brasil, 2010). Observou-se que a redução dos encaminhamentos ocorreu em três etapas: a primeira, como uma consequência imediata do trabalho de apoio matricial, mediante análise de documentos clínicos e sem a presença do paciente na maioria das vezes, baseada em uma relação de confiança entre os profissionais da equipe de referência e os especialistas 
matriciadores; a segunda etapa dependeu diretamente da equipe de referência buscar e reavaliar a necessidade de manter o encaminhamento dos pacientes não priorizados; e a terceira etapa aconteceu em consequência do aumento da competência das equipes de referência, que passaram a indicar com maior precisão os casos que necessitavam da intervenção do especialista, mostrando uma mudança de comportamento dos profissionais.

Com relação à atuação dos cardiologistas, uma análise da oferta de consultas mostrou uma elevada discrepância entre os especialistas, em que alguns ofereciam poucas vagas para novos pacientes e muitas para retornos, enquanto outros devolviam a maioria dos pacientes para as equipes da referência. Um dos onze cardiologistas era responsável por $42 \%$ das consultas de retorno, sendo quase inacessível para consultas novas. O maior problema referido pelas equipes da APS foi a ausência de contrarreferência, conforme se observa nas falas a seguir:

Não existem contrarreferências dos cardiologistas, com uma única exceção

...O que o cardiologista disse? (Médico da APS).

Ahaaaa! Eu não lembro, não sei! (Paciente).

$\varepsilon$ o encaminhamento, você não o trouxe? (Médico da APS).

Ele não devolveu! (Paciente).

Este achado não é peculiaridade da especialidade nem da localidade, tendo sido referido também por Dimenstein e colaboradores (2009), nos serviços de Saúde Mental, em Natal. Se todos os cardiologistas fizerem a contrarreferência, é provável que se reduzam os encaminhamentos, pois a atitude pode agregar conhecimentos e ampliar a capacidade resolutiva da APS. Nenhum especialista pode assegurar uma abordagem integral. É fundamental a adscrição do usuário à equipe de referência, que permanece responsável pelo projeto terapêutico e necessita do apoio do especialista. A ausência de contrarreferência e a absorção da maioria dos pacientes pelos especialistas podem estar reproduzindo atitudes comuns da medicina privada, em que a atividade de clínica médica ou geral está em extinção. Segundo Dimenstein e colaboradores (2009), faz parte de o imaginário social contemporâneo acreditar que a grande especialização profissional, em especial a médica, é a estratégia mais eficiente para se cuidar da saúde. A supervalorização do especialista e o desconhecimento da qualidade do trabalho das equipes da APS estão aparentes no depoimento de cardiologista: não encaminho nenhum paciente para o postinho porque os meus pacientes são todos complexos, graves, e os médicos da rede não vão dar conta, eles não têm competência. Os médicos do PS (aqui no hospital) pedem desesperados para nós atendermos os cardiopatas.

A desvalorização da atenção primária, considerada um local onde se trata apenas patologias simples, é um aspecto já relatado por outros autores (Brasil, 2010), que afirmam ser necessária uma mudança de cultura no SUS, dando centralidade e atribuindo valor para o saber da atenção básica, baseado no vínculo, no seguimento e na construção de um conhecimento singular. A formação inadequada de muitos profissionais de saúde da rede pública é uma constatação já estudada por outros autores (Ceccim, 2005) e que aponta para a necessidade de modificar o currículo dos cursos de graduação da área da saúde e também a seleção e adaptação dos profissionais contratados pelo serviço público. As falas de um médico da APS reforçam este ponto: $\varepsilon$ Eecessário incluir o especialista no processo ao fazê-lo entender que trabalhamos em um Sistema Único de Saúde e trabalhamos em conjunto em prol do paciente.

Além dos profissionais, há necessidade de se mudar a visão dos pacientes, conforme reconhece um dos participantes do programa: Mas tem paciente que exige ser mandado para o especialista e não toma os nossos medicamentos.

O programa de apoio matricial pode favorecer esta mudança no comportamento tanto do profissional quanto do usuário, na medida em que propicia melhorias assistenciais com a qualificação da equipe e dos profissionais de referência e personalização da relação entre a atenção básica e os outros serviços (Campos, 1999; Campos e Domitti, 2007).

Na presente experiência, a discussão dos casos clínicos e os atendimentos e intervenções conjuntas contribuíram para aquisição de competências em Cardiologia, interação dialógica com os cardiologistas e aproximação com a gestão. A atividade de apoio matricial identificou lacunas nos saberes de 
temas da Cardiologia, em que a mais referida foi a interpretação dos laudos dos eletrocardiogramas (ECG), normalmente eu me sinto inseguro com as disritmias, com os bloqueios de ramos, eu fico bastante em dúvida, e justificou um curso presencial para os médicos, com maciço comparecimento dos generalistas. A análise das anormalidades referidas nos laudos dos ECGs, ora inexistentes ora com importância clínica discreta, ou consequente a doenças não cardíacas ou medicamentos, talvez devesse merecer uma melhor abordagem nos cursos de Medicina. A eficácia do tratamento clínico, medicamentoso e não medicamentoso, da doença coronariana também é desconhecida por muitos generalistas, que julgavam necessárias e fundamentais as intervenções cirúrgicas. Aqui também foi necessário desmistificar os riscos e indicar os benefícios dos exercícios físicos. Numerosos profissionais relataram a sua satisfação com a possibilidade de expandir os seus conhecimentos diretamente com o especialista, Olha, se funcionar assim, seria bom ter apoio matricial em endócrino e reumatologia, e solicitaram iniciativas semelhantes em outras especialidades, encabeçadas pela Psiquiatria, Endocrinologia, Reumatologia e Nutrição. Segundo uma enfermeira: outro dia, na reunião de capacitação para a ESF, eu tive uma grande surpresa, os médicos que já passaram pelo apoio matricial se levantaram e começaram a ajudar na reunião, orientando os novos e os outros e comentando que já estavam atuando de acordo com as nossas orientações e que estava dando certo.

Diferente de Dimenstein e colaboradores (2009), que relatam a experiência no apoio matricial em Saúde Mental, aqui havia a percepção da possibilidade do trabalho articulado entre a APS e o serviço especializado. Isto pareceu estar materializado nas queixas da ausência da contrarreferência, na frequente aceitação da responsabilização pelos cuidados de usuários retirados da fila e pela solicitação de corresponsabilização nos cuidados de outros, percebidos pela equipe como casos mais difíceis. A avaliação feita até agora possibilitou identificar que as equipes de referência têm competência para acompanhar de forma resolutiva e responsável muitos dos casos.

Um achado importante foi constatar a carência de equipamentos básicos, tais como os esfigmoma- nômetros de diversos tamanhos. Porém, as requisições conjuntas das equipes de referência e dos profissionais matriciadores resultaram em solução do problema. Outra descoberta que afeta negativamente a atenção na APS foi a elevada rotatividade dos médicos, por transferência para outras unidades ou por encerramento dos contratos provisórios. Aqui se perde o altamente desejado vínculo entre o profissional e a população adscrita à sua unidade. E aqui se reforça a necessidade de continuidade do apoio matricial e de encontros em prazos mais curtos, como tem solicitado as equipes de referência. Em 2012 um cardiologista pediu e foi substituído no Programa de Apoio Matricial. Atualmente o programa expandido abrange as áreas de Psiquiatria, Endocrinologia e Gastroenterologia. Como se trata de uma nova ferramenta, os apoiadores matriciais têm limitações, inclusive no entendimento da proposta, e têm notado que o apoio matricial é maior do que a simples discussão de casos e a redução das "filas" de pacientes para a especialidade. Ao longo do programa um dos apoiadores cancelou muitas reuniões com as equipes e finalmente solicitou a sua saída, por constatar que não tinha o perfil de apoiador. Uma especialista de elevada experiência e longa atuação no SUS, recusou o convite para integrar o Apoio Matricial (AM) alegando ter dificuldades pedagógicas. Por outro lado, um médico docente do curso de Medicina comentou: Quero te dar os parabéns! Os alunos da IBC (Interação Básico Clínica) falaram que te acompanharam no apoio matricial e acharam muito legal, falaram muito bem! Eu não conhecia, nunca ouvi falar de apoio matricial. Precisamos conversar a respeito, para saber como funciona e incluir outras especialidades.

Muitas das reuniões não contaram com a participação de toda a equipe. Os técnicos de enfermagem são os responsáveis pela aferição da pressão arterial e a distribuição dos medicamentos nas unidades, sendo os primeiros interlocutores com os usuários e depositários das suas angústias e necessidades. Devido à sua importância dentro da equipe, especialmente nos cuidados com as DCVs, eles deveriam participar das reuniões, tanto para ampliar os seus conhecimentos quanto para colaborar com preciosas contribuições oriundas de sua experiência cotidiana. Identificaram-se importantes lacunas e 
muitos mitos acerca da hipertensão arterial, cuja persistência resulta em prejuízo dos cuidados com os usuários. Segundo alguns técnicos, Doutor, eu já trabalhei em PA (Pronto Atendimento) e ouvi o médico falar que bom que sangrou o nariz e não lá dentro, e Doutor, isto que o senhor falou pra nóis o senhor falou para os médicos? Porque eles assustam o paciente, querem que a gente coloque o captopril debaixo da língua, mandar para o PA!

A constatação da execução inadequada da técnica de aferição da pressão arterial e da ausência de supervisão pelos enfermeiros e médicos resultou em um curso de atualização em hipertensão dirigido exclusivamente para os técnicos, com a mesma equipe que orientou os enfermeiros e médicos.

\section{Comentários Finais}

O Programa de Apoio Matricial contribuiu para a redução da fila para o cardiologista e permitiu antecipar o atendimento de usuários com problemas mais complexos e/ou graves. Foi desencadeado um processo em que os encaminhamentos realizados pelas equipes de referência se qualificaram, refletindo melhor aptidão e segurança dos profissionais no manejo adequado dos pacientes. Além da qualificação dos profissionais, sua aproximação com a gestão e com os especialistas ajudou a enfrentar vários problemas estruturais. A satisfação de muitos dos participantes, expressa nos depoimentos e nas reuniões de avaliação, com o método e com os resultados, justifica a sua continuidade. A criação de programas de apoio matricial em outras especialidades foi um resultado indireto e evidencia a potencialidade do método em modificar processos de trabalhos na assistência à saúde. Esperam-se entraves ao longo do trabalhoentre especialistas e equipes de referência, cujas zonas de conforto podem gerar inércia e desinteresse com mudanças. A lógica da hierarquização e da transferência de responsabilidade é muito antiga e poderosa para ser subvertida em curto prazo. Portanto, a continuidade do Programa de Apoio Matricial, sua reprodução em outros locais e incorporação de outros profissionais que atuam nas equipes multidisciplinares na atenção primária são maneiras de se conhecer sua potencialidade para produzir mudanças no processo de trabalho das equipes e no controle das DCVs no âmbito do SUS.

\section{Colaboração dos autores}

Todos os autores participaram da concepção e delineamento do estudo, da análise e interpretação dos dados, da redação do artigo ou revisão do texto e da versão final a ser publicada.

\section{Referências}

ARONA, E. C. Implantação do matriciamento nos serviços de saúde de Capivari. Saúde e Sociedade, São Paulo, v. 18, p. 26-36, 2009. Suplemento 1.

BRASIL. Ministério da Saúde. Secretaria de Vigilância em Saúde. Secretaria de Atenção à Saúde. Diretrizes e recomendações para o cuidado integral de doenças crônicas não-transmissíveis: promoção da saúde, vigilância, prevenção e assistência. Brasília, DF, 2006. (Série B, Textos Básicos de Saúde, Série Pactos pela Saúde, v. 8).

BRASIL. Ministério da Saúde. Política Nacional de Humanização. Redes de saúde: uma reflexão sobre formas de gestão e o fortalecimento da atenção básica. In:___. Atenção básica. Brasília, DF, 2010. p. 59-74. (Série B, Textos Básicos de Saúde, Cadernos Humaniza SUS, v. 2).

CAMPBELL, N. R. C. et al. Increases in antihypertensive prescriptions and reductions in cardiovascular events in Canada. Hypertension, Jackson, v. 53, n. 2, p. 128-134, 2009.

CAMPOS, G. W. S. Equipes de referência e apoio especializado matricial: um ensaio sobre a reorganização do trabalho em saúde. Ciência \& Saúde Coletiva, Rio de Janeiro, v. 4, n. 2, p. 393-403, 1999.

CAMPOS, G. W. S.; CUNHA, G. T. Apoio matricial e atenção primária em saúde. Saúde e Sociedade, São Paulo, v. 20, n. 4, p. 961-970, 2011.

CAMPOS, G. W. S.; DOMITTI, A. C. Apoio matricial e equipe de referência: uma metodologia para gestão do trabalho interdisciplinar em saúde. Cadernos de Saúde Pública, Rio de Janeiro, v. 23, n. 2, p. 399-407, 2007. 
CECCIM, R. B. Onde se lê "recursos humanos em saúde", leia-se "coletivos organizados de produção em saúde”: desafios para a educação. In: PINHEIRO, R.; MATTOS, R. A. (Org.). Construção social da demanda: direito à saúde, trabalho em equipe, participação e espaços públicos. Rio de Janeiro: Cepesc, 2005. p. 163-182.

COTTA, R. M. M. et al. Organização do trabalho e perfil dos profissionais do Programa Saúde da Família: um desafio na reestruturação da atenção básica em saúde. Epidemiologia e Serviços de Saúde, Brasília, DF, v. 15, n. 4, p. 7-18, 2006.

DAVIS, D. A. et al. Changing physician performance: a systematic review of the effect of continuing medical education strategies. The Journal of the American Medical Association, Chicago, v. 274, n. 9, p. 700-708, 1995.

DIMENSTEIN, M. et al. O apoio matricial em Unidades da Saúde da Família: experimentando inovações em saúde mental. Saúde e Sociedade, São Paulo, v. 18, n. 1, p. 63-74, 2009.

FIGUEIREDO, M. D.; CAMPOS, R. O. Saúde mental na atenção básica à saúde de Campinas, SP: uma rede ou um emaranhado? Ciência \& Saúde Coletiva, Rio de Janeiro, v. 14, n. 1, p. 129-138, 2009.

HOEPFNER, C. Controle dos níveis pressóricos nos portadores de hipertensão arterial atendidos nas unidades básicas de saúde do município de Joinville. 2010. Dissertação (Mestrado de Saúde e Meio Ambiente) - Universidade da Região de Joinville, Joinville, 2010.

HOEPFNER, C.; FRANCO, S. C. Inércia clínica e controle da hipertensão arterial nas unidades de atenção primária à saúde. Arquivos Brasileiros de Cardiologia, Rio de Janeiro, v. 95, n. 2, p. 223-229, 2010.
JOINVILLE (Santa Catarina). Secretaria Municipal de Saúde. Protocolo de hipertensão arterial sistêmica. Joinville, 2007.

JOINVILLE (Santa Catarina). Secretaria Municipal da Saúde. Linhas-guia da atenção básica: hipertensão arterial. Joinville, 2010.

LEMOS, S. M. L. et al. Utilização de diretrizes clínicas e resultados na atenção básica à hipertensão arterial. Cadernos de Saúde Pública, Rio de Janeiro, v. 25, n. 9, p. 2001-2011, 2009.

McALISTER, F. A. The Canadian Hypertension Education Program: a unique Canadian initiative. Canadian Journal of Cardiology, Montreal, v. 22, n. 7, p. 559-564, 2006.

MILCHAK, J. L. et al. Measuring adherence to practice guidelines for the management of hypertension. Hypertension, Jackson, v. 44, n. 5, p. 602-608, 2004.

MION, J. R. D. et al. Os médicos brasileiros seguem as diretrizes brasileiras de hipertensão? Arquivos Brasileiros de Cardiologia, Rio de Janeiro, v. 88, n. 2, p. 212-217, 2007.

MORAIS, A. P. P.; TANAKA, O. Y. Apoio matricial em saúde mental: alcances e limites na atenção básica. Saúde e Sociedade, São Paulo, v. 21, n. 1, p. 161-170, 2012.

ONYSKO, J. et al. Large increase in hypertension diagnosis and treatment in Canada after a healthcare professional education program. Hypertension, Jackson, v. 48, n. 5, p. 853-86o, 2006.

THIOLLENT, M. J. M. Metodologia da pesquisaação. 18. ed. São Paulo: Cortez, 2011. 